\title{
Two-step flux penetration in layered antiferromagnetic superconductor
}

\author{
T. Krzysztoń \\ Institute of Low Temperature and Structure Research, Polish Academy of Sciences, \\ 50-950 Wroctaw, P.O.Box 1410, Poland
}

\begin{abstract}
A layered antiferromagnetic superconductor in the mixed state may posses magnetic domains created along the Josephson vortices. This may happen when an external magnetic field is strong enough to flip over magnetic moments, lying in the phase core of the Josephson vortex, from their ground state configuration. The formation of the domain structure of the vortices modifies the surface energy barrier of the superconductor. During this process the entrance of the flux is stopped and a newly created state exhibits perfect shielding. Such behavior should be visible as a plateau on the dependence of flux density as a function of the external magnetic field. The end of the plateau determines the critical field, which we call the second critical field for flux penetration.
\end{abstract}

Key words: High- $T_{C}$ superconductivity, mixed state, magnetic superconductors PACS: 74.60.Ec, 74.72.-h

\section{INTRODUCTION}

Among classical magnetic superconductors there are three groups of cluster compounds, $\mathrm{REMo}_{6} \mathrm{~S}_{8}, \mathrm{REMo}_{6} \mathrm{Se}_{8}$, and $\mathrm{RERh}_{4} \mathrm{~B}_{4}(\mathrm{RE}=$ rare-earth) which have been the primary systems for study of the interplay between superconductivity and long-range magnetic order [1,2]. Although good quality single crystals of these materials have been available and measured for a long time a very interesting phenomenon was recently discovered in $\mathrm{DyMo}_{6} \mathrm{~S}_{8}$ due to very carefully conducted experiment [3]. This phenomenon predicted in [4] and named two-step flux penetration was previously observed solely on (bct) $\mathrm{ErRh}_{4} \mathrm{~B}_{4}$ [5]. The present work is inspired by this discovery and the hope that the same behavior could possibly be observed in some of the layered superconducting structures. The specific feature caused by the long antiferromagnetic order in the mixed state of a superconductor is the possibility of creation of 
the spin-flop (SF)(or metamagnetic) domains along the vortices [6]. This is easy to understand by taking two sublattices antiferromagnet as an example. An infinitesimal magnetic field applied perpendicular to the easy axis makes the ground antiferromagnetic (AF) state unstable against the phase transformation to the canted phase $(\mathrm{SF})$. On the contrary, if the magnetic field is applied parallel to the easy axis the antiferromagnetic configuration is stable up to the thermodynamic critical field $H_{T}$. When the field is further increased a canted phase develops in the system. Let us assume that in an antiferromagnetic superconductor the lower critical field fulfils the relation $H_{c 1}<\frac{1}{2} H_{T}$ and that the external field, $H_{c 1}<H<\frac{1}{2} H_{T}$, is applied parallel to the easy axis. Then the superconducting vortices appear in the ground antiferromagnetic state. When the field is increased above $H_{p l}$ (see Fig 3) approximately equal to $\frac{1}{2} H_{T}$ the phase transition to the canted phase originates in the vortex core. The spatial distribution of the field around the vortex is a decreasing function of the distance from its center. Hence the magnetic field intensity in the neighborhood of the core is less then $H_{T}$. Therefore, the rest of the vortex remains in the antiferromagnetic configuration. The radius of the SF domain grows as the field is increased. The above considerations apply to the classical superconducting Chevrel phases as well as to the high $T_{c}$ superconductors, where antiferromagnetic order is produced by the regular lattice of RE ions occupying isolating layers. In this paper we consider the structure shown on

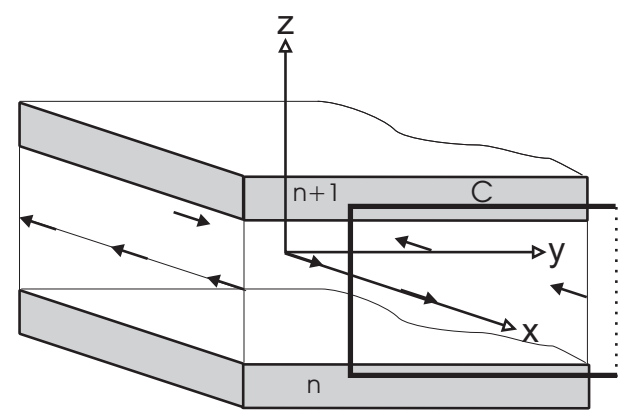

Fig. 1. Schematic drawing of a piece of the layered superconductor. The shaded areas $(n, n+1)$ represent superconducting layers. The bold arrows represent magnetic moments of RE ions lying in the isolating layers. The axes of the reference frame are shown.

Fig. 1 that we believe simulates a real structure of many antiferromagnetic layered superconductors. A good candidate to show the above behavior should possess the isolating layers with the magnetic moments of RE ions running parallel and antiparallel to the direction (easy axis) lying in the $a b$ plane. A typical example of such system is $\mathrm{ErBa}_{2} \mathrm{Cu}_{3} \mathrm{O}_{7}$. This compound has tetragonal unit cell with small orthorombic distortion in the $a b$ plane. The Er ions form two sublattices antiferromagnetic structure of magnetic moments laying parallel and antiparallel to the $b$ direction [7]. Another example may be Er nickel boride-carbides 8,9,10. The layered structure of RE nickel boride-carbides is reminiscent of that of the high- $T_{c}$ oxide superconductors and consists of 
RE-carbon layers separated by $\mathrm{Ni}_{2} \mathrm{~B}_{2}$ sheets.

\section{BASIC EQUATIONS}

We start description of our problem in terms of the Lawrence-Doniach energy functional. In this approach a layered superconductor is described by the superconducting planes with the interlayer distance d, as shown on Fig. 1. The antiferromagnetic subsystem consisting of RE ions is confined to the insulating layers. The magnetic moments are running parallel and antiparallel to the $\mathrm{x}$-axis (easy axis). The Lawrence-Doniach functional is obtained from the standard Ginzburg-Landau energy by discretization of the kinetic energy in the z-direction.

$$
\begin{array}{r}
F_{S}=\int d^{2} r d \sum_{n}\left[\frac{\hbar^{2}}{2 m}\left|\left(-i \nabla_{(\mathbf{2})}+\frac{2 i e}{\hbar} \mathbf{A}_{(\mathbf{2})}\right) \Psi_{n}\right|^{2}+a\left|\Psi_{n}\right|^{2}+\frac{1}{2} b\left|\Psi_{n}\right|^{4}\right. \\
\left.+\frac{\hbar^{2}}{2 \mathcal{M} d^{2}}\left|\Psi_{n+1} \exp \left(\frac{2 e i}{\hbar} \int_{n d}^{(n+1) d} A_{z} d z\right)-\Psi_{n}\right|^{2}\right]
\end{array}
$$

The quantity $\hbar, e, m$, denote Planck constant, charge of the electron and mass of the current carrier in the $a b$ plane, whereas $\mathcal{M}$ denotes mass of the current carrier in the z-direction. The antiferromagnetic two sublattices subsystem with single ion anisotropy is described with the following energy density functional

$$
f_{M}=\sum_{n}\left\{J \mathbf{M}_{1 n} \cdot \mathbf{M}_{2 n}+K \sum_{i=1}^{2}\left(M_{i n}^{x}\right)^{2}-|\gamma| \sum_{i=1}^{2} \sum_{j=x, y, z}\left(\nabla M_{i n}^{j}\right)^{2}\right\} .
$$

where $\mathbf{M}_{n}=\mathbf{M}_{1 n}+\mathbf{M}_{2 n}$ is the sum of the magnetization vectors of the sublattices in the n-th insulating layer, $M_{i n}^{x}$ is the component of the magnetization sublattice vector along the anisotropy axis in the n-th layer, $J$ denotes the exchange constant between two sublattices, $K$ is the single ion anisotropy constant, $\sqrt{|\gamma|}$ is the magnetic stiffness length, and $M_{0}=\left|\mathbf{M}_{1 n}\right|=\left|\mathbf{M}_{2 n}\right|$. Since in the following we analyze the phenomena with characteristic lengthscales much larger then the interatomic distance it is justified to omit the gradient term in $f_{M}$. The components of the total magnetization vector $\mathbf{M}$ have the following form in both sublattices: $M_{i y}=M_{0} \sin \theta_{i}, M_{i x}=M_{0} \cos \theta_{i}$, where $\theta_{i}$ (canted spin angle) is the angle between the magnetization in the sublattice and the external magnetic field directed along the $x$-axis. The AF $\left(\theta_{1}=0, \theta_{2}=\pi\right)$ and SF phases $\left(\theta_{1}=-\theta_{2}=\theta\right)$ are in thermodynamic equilibrium in an applied field equal to the thermodynamic critical field [11]

$$
H_{T}=M_{0}[K(J-K)]^{1 / 2} .
$$


The canted spin angle of the SF phase is then expressed as

$$
\cos \theta=\frac{K M_{0}}{H_{T}} .
$$

Finally we add the magnetic field energy to obtain the free energy of the entire system

$$
F=F_{s}+\int\left\{f_{M}+\frac{\mu_{0}}{2} \mathbf{H}^{2}\right\} d V .
$$

According to experiments the antiferromagnetic order is very weak affected by the presence of superconductivity, then it is reasonable to neglect the effect of superconductivity on the exchange interaction in $F$. Instead we introduce electromagnetic coupling between the magnetic and superconducting subsystem. This means that both order parameters $\Psi_{n}$ and $\mathbf{M}$ are coupled through the vector potential $\mathbf{A}$

$$
\begin{gathered}
\mathbf{B}=\operatorname{rot} \mathbf{A}=\mu_{0} \mathbf{H}+\mathbf{M}, \\
\boldsymbol{j}_{s}=\operatorname{rot} \mathbf{H},
\end{gathered}
$$

where $\mathbf{B}$ is the vector of the magnetic flux density (magnetic induction) and $\mathbf{H}$ is the vector of the thermodynamic magnetic field intensity. The functional (5) can be treated in the London approximation by assuming a constant modulus $\Psi_{n}$ within the planes and allowing only for phase $\left(\varphi_{n}\right)$ degree of freedom. The equilibrium conditions of the whole system can be obtained via minimization the Gibbs free energy functional $G=F-\int\left(\mathbf{B H}_{0}\right) d V$. Performing this task with respect to vector potential $\mathbf{A}$ and $\varphi_{n}$ provides us with the fundamental equations for currents and phases.

$$
\begin{gathered}
\sum_{n} \frac{d}{\lambda^{2}}\left(\frac{\phi_{0}}{2 \pi} \nabla_{(\mathbf{2})} \varphi_{n}-\mathbf{A}_{(\mathbf{2})}\right) \delta(z-n d)=\mu_{0} \mathbf{j}_{(\mathbf{2})}=\operatorname{rot}_{(2)}(\mathbf{B}-\mathbf{M}) \\
\sum_{n}\left(\frac{\phi_{0}}{2 \pi} \frac{1}{\lambda_{j}^{2} d} \sin \chi_{n+1, n}\right) \Theta(z-d n) \Theta[d(n+1)-z]=\mu_{0} j_{z}=\operatorname{rot}_{z}(\mathbf{B}-\mathbf{M}) \\
\nabla_{(\mathbf{2})}\left(\nabla_{(\mathbf{2})} \varphi_{n}-\frac{2 \pi}{\phi_{0}} \mathbf{A}_{(\mathbf{2})}\right)=\frac{1}{r_{j}^{2}}\left(\sin \chi_{n+1, n}-\sin \chi_{n, n-1}\right)
\end{gathered}
$$

where $\delta(z-n d)$ is the Dirac delta function, $\Theta(z-d n)$ Heaviside step function, $\lambda$ denotes London penetration depth in the superconducting plane, $\lambda_{j}=$ $\lambda \sqrt{\mathcal{M} / m}, r_{j}=d \sqrt{\mathcal{M} / m}$ and $\chi_{n+1, n}=\varphi_{n+1}-\varphi_{n}+\frac{2 e i}{\hbar} \int_{n d}^{(n+1) d} A_{z} d z$ is the gauge invariant phase difference. In the following we shall investigate the problem of a single vortex line lying parallel to the Josephson coupled superconducting layers, separated by the insulating antiferromagnetic layers. 


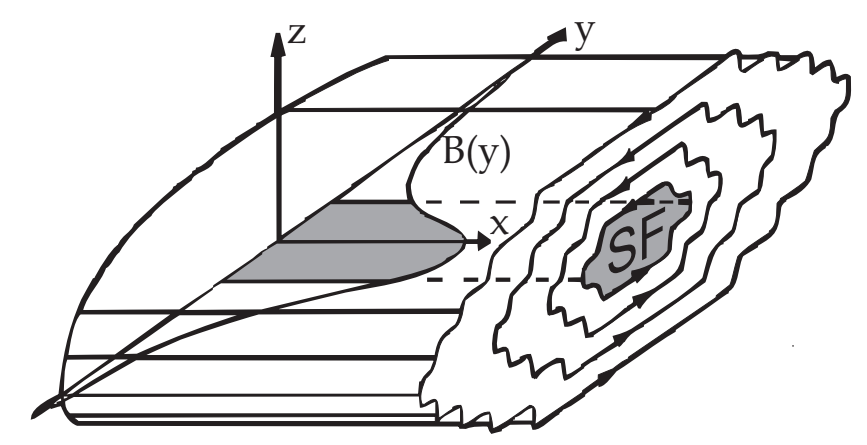

Fig. 2. Single Josephson vortex lying in the $a b$ plane along the $\hat{x}$-axis. The shaded area shows induced SF domain along the phase core.

\section{SINGLE JOSEPHSON VORTEX}

We assume that the vortex center, located in the central $n=0$ layer, is parallel to the $x$-axis. The relation between the magnetic field of the vortex and the gauge-invariant phase difference $\chi_{n+1, n}(y)$ of the superconducting wave function across layers $n$ and $n+1$ can be found by integrating the vector potential given by equations (8) and (9) around a rectangular, semi-infinite contour C, as shown in Fig. 1. This contour, parallel to the $y z$ plane, is located apart from the central junction $n=0$, where nonlinearities of the phase difference must be taken into account. The magnetic flux within this contour in given as.

$$
\Phi(y)=d \int_{y}^{\infty} d y^{\prime} B\left(y^{\prime}, z\right)=\oint_{C} \mathbf{A} d \boldsymbol{l}
$$

Differentiating the result with respect to $y$ one obtains

$$
\mathbf{B}=\lambda^{2} \frac{\partial^{2}(\mathbf{B}-\mathbf{M})}{\partial z^{2}}+\lambda_{j}^{2} \frac{\partial^{2}(\mathbf{B}-\mathbf{M})}{\partial y^{2}}
$$

The above equation shows that in the Josephson vortex the screening currents vanish on a length scale $\lambda_{j}$ along $y$ axis, and a scale $\lambda$ along $z$ direction. On these scales the Josephson and Abrikosov vortices in an anisotropic superconductor are roughly equivalent apart from small corrections in the current flow, Fig. 2, and the magnetic field pattern due to the layered structure. But in contrast to the Abrikosov vortex, where the large current flow near the core leads to complete supression of the order parameter, the supression of the order parameter in the superconducting layers is only weak in the so called phase core of the Josephson vortex. Within the distances $r_{j}$ along $y$, and $d$ along $z$ we have to take into account the nonlinearity and discretness of Eq. (10). On these length scales the phase is changing rapidly and the current density $j_{z}$ reaches its maximum value $j_{c}=\frac{\phi_{0}}{2 \pi \mu_{0} \lambda_{j}^{2} d}$. In the region of the phase core London model fails.

To make the problem simpler we assume that the magnetization in the isolated 
vortex is constant across the SF phase domain. Thus, we can write

$$
|\mathbf{M}|=\left\{\begin{array}{cl}
M & \text { if } \rho \leq \rho_{m} \\
0 & \text { if } \rho>\rho_{m}
\end{array},\right.
$$

where $\rho_{m}$ is dimensionless radius of the $S F$ domain in the cylindrical reference frame $x=x ; y=\lambda_{j} \rho \sin \theta ; z=\lambda \rho \sin \theta$. Then the solution of Eq. (11) for a single Josephson vortex is given by the modified Bessel functions $K_{0}$ and $I_{0}$

$$
\begin{aligned}
b_{\mathrm{SF}} & =C_{1} K_{0}(\rho)+C_{2} I_{0}(\rho), \text { for } \rho_{j}<\rho \leq \rho_{m} \\
b_{\mathrm{AF}} & =C_{3} K_{0}(\rho), \text { for } \rho>\rho_{m},
\end{aligned}
$$

( $\rho_{j}$ denotes the dimensionless phase coherence length ) with the following boundary conditions:

$$
\begin{aligned}
b_{\mathrm{SF}}\left(\rho_{m}\right) & =\mu_{0} H_{T}+M=B_{T} \\
b_{\mathrm{AF}}\left(\rho_{m}\right) & =\mu_{0} H_{T} .
\end{aligned}
$$

These conditions, together with the flux quantization condition, are used to calculate the arbitrary constants in Eq. (13).

$$
\begin{aligned}
C_{1}= & \frac{B_{T} \rho_{m} I_{1}\left(\rho_{m}\right)-\left[\mu_{0} H_{T} \rho_{m} \frac{K_{1}\left(\rho_{m}\right)}{K_{0}\left(\rho_{m}\right)}-\frac{\varphi_{0}}{2 \pi \lambda \lambda_{j}}\right] I_{0}\left(\rho_{m}\right)}{\rho_{m} K_{1}\left(\rho_{m}\right) I_{0}\left(\rho_{m}\right)-I_{0}\left(\rho_{m}\right)+\rho_{m} K_{0}\left(\rho_{m}\right) I_{1}\left(\rho_{m}\right)} \\
C_{2}= & \frac{B_{T}\left[\rho_{m} K_{1}\left(\rho_{m}\right)-1\right]+\left[\mu_{0} H_{T} \rho_{m} \frac{K_{1}\left(\rho_{m}\right)}{K_{0}\left(\rho_{m}\right)}-\frac{\varphi_{0}}{2 \pi \lambda \lambda_{j}}\right] K_{0}\left(\rho_{m}\right)}{\rho_{m} K_{1}\left(\rho_{m}\right) I_{0}\left(\rho_{m}\right)-I_{0}\left(\rho_{m}\right)+\rho_{m} K_{0}\left(\rho_{m}\right) I_{1}\left(\rho_{m}\right)} \\
C_{3}= & \frac{\mu_{0} H_{T}}{K_{0}\left(\rho_{m}\right)} .
\end{aligned}
$$

Finally we write free energy of the isolated vortex

$$
\begin{aligned}
\varepsilon & =\frac{\lambda_{j} \lambda}{2 \mu_{0}} \oint_{\sigma_{1}} d \boldsymbol{\sigma}\left\{\left[\mathbf{b}_{\mathrm{SF}}(\boldsymbol{r})-\mathbf{M}\right] \times \operatorname{rot}_{\mathrm{SF}}(\boldsymbol{r})\right\} \\
& +\frac{\lambda_{j} \lambda}{2 \mu_{0}} \oint_{\sigma_{2}} d \boldsymbol{\sigma}\left\{\mathbf{b}_{\mathrm{AF}}(\boldsymbol{r}) \times \operatorname{rot}_{\mathrm{AF}}(\boldsymbol{r})\right\}
\end{aligned}
$$

where $\boldsymbol{r}=\left(\frac{y}{\lambda_{j}}, \frac{z}{\lambda}\right)$ is the position of the vortex line, $\sigma_{1}$ denotes the surface of the phase core, and $\sigma_{2}$ the surface of the SF domain respectively. The integrals in Eq. (16) performed as line integrals along the contours of the cross 
sections of the appropriate surfaces give $\varepsilon_{1}$ - the line tension of the vortex. The minimum of $\varepsilon_{1}$ with respect to $\rho_{m}$ determines

$$
\rho_{m}^{2}=\frac{5 \phi_{0}}{8 \pi \lambda \lambda_{j} B_{T}}
$$

\section{FREE ENERGY OF THE LATTICE}

Equation (11), in the new coordinates, can be rewritten for the lattice of vortices in the following way:

$$
\mathbf{B}+\operatorname{rotrot} \mathbf{B}=\frac{\phi_{0}}{\lambda \lambda_{j}} \sum_{m} \delta\left(\boldsymbol{r}-\boldsymbol{r}_{m}\right),
$$

where $r_{m}$ specify the positions of the phase cores of the vortices. The solution of Eq. (18) is then a superposition

$$
\mathbf{B}(\boldsymbol{r})=\sum_{m} \mathbf{B}_{m}\left(\boldsymbol{r}-\boldsymbol{r}_{m}\right)
$$

of the solutions $\mathbf{B}_{m}\left(\boldsymbol{r}-\boldsymbol{r}_{m}\right)$ of isolated vortices at points $\boldsymbol{r}_{m}$. The free energy of the system can thus be written as

$$
F=\frac{\lambda \lambda_{j}}{2 \mu_{0}} \oint_{\sigma} d \boldsymbol{\sigma}(\mathbf{B} \times \operatorname{rot} \mathbf{B})
$$

The above symbolic surface integral is taken over the surfaces of the phase cores and surfaces of the SF domains. The energy of the Meissner state is chosen as zero of the energy scale. Again, when the surface integrals are replaced by contour ones over appropriate cross sections we get line energy of the system. This, in turn, multiplied by vortex density $n$ gives $f$-free energy density of the system. After some transformations one can derive the following formula

$$
\begin{gathered}
f=n \varepsilon_{1}+n \phi_{0} H_{T}(\ln \beta)^{-1} \sum_{m} K_{0}\left(r_{m}\right), \\
\beta=\sqrt{\frac{\pi \lambda \lambda_{j} B_{T}}{\phi_{0}}},
\end{gathered}
$$

here the sum is over all vortices excluding the one in the origin, and $r_{m}$ denotes the distance of a vortex from the origin. The lattice sum may now be replaced by integral in the $y z$-plane over a smoothed vortex density, excluding the area

$n^{-1}$ associated with the single flux line in the origin. The free energy density then reduces to

$$
f=n \varepsilon_{1}+B^{2}\left(\frac{H_{T}}{B_{T}}\right)\left(\frac{\beta}{\ln \beta}\right)+B \frac{H_{T}}{4 \ln \beta} \sqrt{\frac{4 \lambda_{j}}{27 \lambda}} \ln \left(\frac{a}{\sqrt{\lambda \lambda_{j}}}\right)
$$




$$
\left(\frac{a}{\sqrt{\lambda \lambda_{j}}}\right)^{2}=\frac{1}{\beta^{2}}\left(\frac{B_{T}}{B}\right) \sqrt{\frac{4 \lambda_{j}}{27 \lambda}}
$$

here $a=\left|\boldsymbol{a}_{1}\right|$ denotes the length of the basal vector of the nonequilateral triangular unit cell, and $2\left|\boldsymbol{a}_{2}\right|=a \sqrt{1+\tan ^{2} \alpha}$ ( $\alpha$ is the angle between both vectors), $\tan \alpha=\sqrt{\frac{3 \lambda}{\lambda_{j}}}[12]$. To determine the equilibrium state it is necessary to minimize the Gibbs free energy density with respect to magnetic induction. The result yields an implicit equation for the constitutive relation $B=B(H)$

$$
H-\frac{\varepsilon_{1}}{\phi_{0}}=B\left(\frac{H_{T}}{B_{T}}\right)\left(\frac{2 \beta}{\ln \beta}\right)+\frac{H_{T}}{4 \ln \beta} \sqrt{\frac{4 \lambda_{j}}{27 \lambda}} \ln \left(\frac{a}{\sqrt{\lambda \lambda_{j}}}\right)
$$

\section{FLUX PENETRATION}

Consider semi-infinite specimen in the half space $y \geq 0$, the vortex and the external magnetic field running parallel to the surface in the $x$ direction. The presence of a surface of the superconductor leads to a distortion of the field and current of any vortex located within a distance of the order of penetration depth from the surface. To fulfill the requirement that the currents cannot flow across the surface of the superconductor we need to introduce an image vortex, with vorticity opposite to the real one. Both vortices, direct and image, interact as real ones except that the interaction is attractive. In the low flux density regime, Clem [13] has shown that there exist two regions: a vortex-free region of the width $y_{f f}$ near the surface of the sample, and a constant flux density region for $y>y_{f f}$. Within the vortex-free area one can introduce the locally averaged magnetic field $B_{M}$ which is a linear superposition of the Meissner screening field, the averaged direct vortices flux density exponentially decreasing towards the surface from its interior value $B$ at $y=y_{f f}$, and averaged image vortices flux density. In our problem the $x$ component of this superposition can be approximated by

$$
B_{M}=B \cosh \left(\frac{y_{f f}-y}{\lambda_{j}}\right) .
$$

The boundary condition $B_{M}(0)=\mu_{0} H_{0}$ determines the thickness of the vortex-free region

$$
y_{f f}=\lambda_{j} \cosh ^{-1}\left(\frac{\mu_{0} H_{0}}{B}\right) .
$$

We assume that the test vortex line is lying within vortex free region at a point $\boldsymbol{r}=\left(\frac{y}{\lambda_{j}}, 0\right)$, and its image at $\boldsymbol{r}=\left(-\frac{y}{\lambda_{j}}, 0\right)$ outside the superconductor. Now the local field of the test vortex can be understood as a superposition of the following fields 


$$
\begin{aligned}
\mathbf{B}_{\mathrm{SF}} & =\mathbf{b}_{\mathrm{SF}}(\boldsymbol{r})-\mathbf{b}_{\mathrm{AF}}(2 \boldsymbol{r})+\hat{x} B_{M}\left(\boldsymbol{r}_{f f}-\boldsymbol{r}\right) \\
\mathbf{B}_{\mathrm{AF}} & =\mathbf{b}_{\mathrm{AF}}(\boldsymbol{r})-\mathbf{b}_{\mathrm{AF}}(2 \boldsymbol{r})+\hat{x} B_{M}\left(\boldsymbol{r}_{f f}-\boldsymbol{r}\right),
\end{aligned}
$$

where $\boldsymbol{r}_{f f}=\left(\frac{y_{f f}}{\lambda_{j}}, 0\right)$, and $\hat{x}$ denotes the unit vector in the $x$ direction. Having determined the local magnetic field we can write the Gibbs free energy of the test vortex line as

$$
\begin{aligned}
G & =\frac{\lambda \lambda_{j}}{2 \mu_{0}} \oint_{\sigma_{1}} d \boldsymbol{\sigma}\left\{\left[\mathbf{B}_{\mathrm{SF}}(\boldsymbol{r})-2 \mu_{0} \mathbf{H}_{0}-\mathbf{M}\right] \times \operatorname{rot} \mathbf{B}_{\mathrm{SF}}(\boldsymbol{r})\right\} \\
& +\frac{\lambda \lambda_{j}}{2 \mu_{0}} \oint_{\sigma_{2}} d \boldsymbol{\sigma}\left\{\left[\mathbf{B}_{\mathrm{AF}}(\boldsymbol{r})-2 \mu_{0} \mathbf{H}_{0}\right] \times \operatorname{rot} \mathbf{B}_{\mathrm{AF}}(\boldsymbol{r})\right\} \\
& +\frac{\lambda \lambda_{j}}{2 \mu_{0}} \oint_{\sigma_{2}} d \boldsymbol{\sigma}\left\{\hat{x} B_{M}\left(\boldsymbol{r}_{f f}-\boldsymbol{r}\right) \times \operatorname{rot} \mathbf{B}_{\mathrm{AF}}(\boldsymbol{r})\right\}
\end{aligned}
$$

After some transformations [13,14] one can obtain the Gibbs free energy per unit length $\mathcal{G}$

$$
\mathcal{G}=\mathcal{G}_{1}+\mathcal{G}_{1}^{\prime}+\mathcal{G}_{\mathcal{M}}
$$

where

$$
\begin{aligned}
\mathcal{G}_{1} & =\varepsilon_{1}-\frac{\lambda \lambda_{j} \pi}{4 \mu_{0}} D_{1} b_{\mathrm{AF}}(2 r) \\
\mathcal{G}_{1}^{\prime} & =-\frac{\lambda \lambda_{j} \pi}{2 \mu_{0}} D_{1}\left[b_{\mathrm{AF}}\left(r_{f f}\right)-b_{\mathrm{AF}}\left(r_{f f}+r\right)\right] \\
\mathcal{G}_{\mathcal{M}} & =-\frac{\lambda \lambda_{j} \pi}{2 \mu_{0}}\left[D_{1} \mu_{0} H_{0}-D_{2} B_{M}\left(r_{f f}-r\right)\right],
\end{aligned}
$$

and

$$
\begin{aligned}
& D_{1}=-\left.\rho_{j} \frac{d b_{\mathrm{SF}}(\rho)}{d \rho}\right|_{\rho=\rho_{j}}-\left.\rho_{m} \frac{d b_{\mathrm{SF}}(\rho)}{d \rho}\right|_{\rho=\rho_{m}}-\left.\rho_{m} \frac{d b_{\mathrm{AF}}(\rho)}{d \rho}\right|_{\rho=\rho_{m}} \\
& D_{2}=-\left.\rho_{j} \frac{d b_{\mathrm{SF}}(\rho)}{d \rho}\right|_{\rho=\rho_{j}}-\left.\rho_{m} \frac{d b_{\mathrm{SF}}(\rho)}{d \rho}\right|_{\rho=\rho_{m}}-\left.2 \rho_{m} \frac{d b_{\mathrm{AF}}(\rho)}{d \rho}\right|_{\rho=\rho_{m}}
\end{aligned}
$$

$\mathcal{G}_{1}$ describes the interaction of the test vortex with its image, $\mathcal{G}_{1}^{\prime}$ is a correction term introduced by Clem [13], and $\mathcal{G}_{\mathcal{M}}$ describes the interaction energy of the test vortex with the modified Meissner field. To find the conditions of the vortex entrance and exit, one has to solve a force balance equation for the test vortex, at the surface of the sample, and at the edge of the flux-filled area, respectively. A calculation using $\mathcal{G}_{1}$ and $\mathcal{G}_{\mathcal{M}}$ alone gives non vanishing force on the test vortex at $\boldsymbol{r}=\boldsymbol{r}_{f f}$. However, the force should be zero there, because $\mathcal{G}_{\mathcal{M}}$ is supposed to account for all the image vortices. To avoid double counting the image vortex one can subtract from the self-energy a contribution of the 
excess image fixed at $\boldsymbol{r}=-\boldsymbol{r}_{f f}$. One can easily check that $\mathcal{G}_{1}^{\prime}$ is negligible at the surface of the sample and has no influence on the conditions of the flux entrance. When the flux starts to enter the sample, $H_{0}=H_{e n 2}(B)$,

$$
y_{f f}=y_{e n}=\lambda_{j} \cosh ^{-1}\left(\frac{\mu_{0} H_{e n 2}(B)}{B}\right) \text {, }
$$

and the energy barrier is moved toward the surface within $\rho_{m}$. Thus, one can derive from the force balance equation

$$
-\left.\frac{D_{1}}{2 D_{2}} \frac{d b_{\mathrm{AF}}(\rho)}{d \rho}\right|_{\rho=\rho_{m}}=B \sinh \left(\frac{y_{e n}}{\lambda_{j}}\right) .
$$

The left hand side of the above equation gives $H_{e n 2}(0)=H_{T} \beta(2 \ln \beta)^{-1}$. This field may be thought as the second critical field for flux penetration calculated in the single vortex approximation [14. Combining Eqs. (30) and (31) we finally obtain

$$
H_{e n 2}(B)=\sqrt{B^{2}+\left(\frac{\mu_{0} H_{T} \beta}{2 \ln \beta}\right)^{2}} .
$$

In the opposite case, when the flux exits the sample, the surface energy barrier tends to the edge of the flux-filled zone. Similar considerations as the above show that

$$
\mu_{0} H_{e x 2}(B) \simeq B
$$

The measure of the height of the energy barrier against flux entrance is

$$
\Delta H_{e n}(B)=\left|H_{e n 2}(B)-H_{e q}(B)\right|,
$$

and against flux exit

$$
\Delta H_{e x}(B)=\left|H_{e q}(B)-H_{e x 2}(B)\right|,
$$

where $H_{e q}$ is given by Eq. (22).

\section{DISCUSSION OF THE RESULTS}

Let us make a short summary of the calculations and visualize the results on schematic magnetization curve shown in the Fig. 3. When the external field is not strong enough to create the SF domains inside vortices, than the magnetization process of the sample being entirely in the AF phase is as follows. The vortices without magnetic structure start to enter the specimen at $H_{e n 1}$. When the field is increased up to the value $H_{p l}$, which is of the order of $H_{T}$, the SF domains are created. Now, the screening current must redistribute its flow in order to keep constant the flux carried by the vortex. This feature is easily seen from Eqs.(13-15). The redistribution of the screening current 


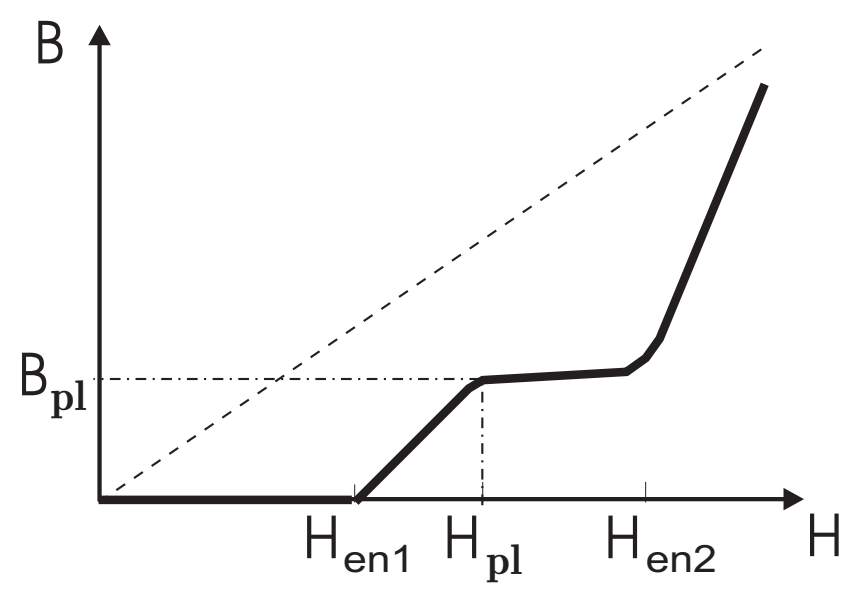

Fig. 3. Schematic drawing of the magnetization process. $H_{e n 1}$ denotes the first penetration field for vortices without magnetic structure, $H_{p l}$ is the applied field which originates SF transitions inside vortices, and $B_{p l}$ is the corresponding flux density. $H_{e n 2}$ is the entrance field for the vortices possessing magnetic structure.

changes the surface energy barrier preventing vortices from entering the sample as expressed in Eq. (28). It means that the density of vortices $n$ is kept constant. Consequently the averaged flux density in the sample $B=n \varphi_{0}$ remains constant when the external field is increased. In Fig. 3 this feature is visible as a plateau on the $B(H)$ curve, or alternatively as a second negative slope on the $M(H)$ curve. The vortices start to penetrate the sample when the external field reaches the right edge of the plateau. We call this value, given by Eq. (32), second critical field for flux penetration $H_{e n 2}$.

To find the thermodynamic critical field $H_{T}$, and then to calculate $H_{e n 2}(B)$ the following argumentation is proposed. At low fields, in the vicinity of the lower critical field $H_{c 1}$, the intensity of the field in the vortex core is $2 H_{c 1}$ [15]. When the external field is increased the field intensity in the vortex core increases because of the superposition of the fields of the surrounding vortices. The field intensity in the core must reach $H_{T}$ in order to originate a transition to the SF phase. Thus, taking into account only the nearest neighbors we can write for the nonunilateral triangular lattice

$$
H_{T}=2 H_{c 1}+z \frac{\varphi_{0}}{\pi \lambda \lambda_{j} \mu_{0}}\left[K_{0}\left(\frac{a}{\lambda_{j}}\right)+2 K_{0}\left(\frac{a}{2 \lambda_{j}} \sqrt{\frac{3 \lambda}{\lambda_{j}}}\right)\right]
$$

here $a$ corresponds to the value $B_{p l}$ of the flux density for which the penetration process stops, see Fig. (3). From the relation $B_{p l}=2 \varphi_{o} \sqrt{\lambda_{j}} /\left(a^{2} \sqrt{3 \lambda}\right)$ one can compute $a$, which in turn may be inserted back into Eq. (34). It is easy to estimate the saturation magnetization $M_{0}$ taking into account the volume of the elementary cell. Then, Eqs. (3) and (4) can be used to calculate $M$ in the 
SF-phase domain

$$
M=2 M_{0} \cos \theta=\frac{2 K M_{0}^{2}}{H_{T}}
$$

\section{CONCLUSION}

The layered antiferromagnetic superconductor may reveal below $T_{N}$ a very interesting behavior in the magnetic field applied parallel to the superconducting planes. When the sample is in the virgin state, initially it magnetizes like ordinary type II superconductor. Upon the applied magnetic field of intensity equal to the critical field for flux penetration the sample undergoes a transformation from the Meissner to the mixed state. Then, the magnetization may proceed in an unusual way. When the field is further increased a new state may appear in which vortices possesses the spin-flop phases created around the cores. We have assumed that in this new state vortices undergo metamorphosis to the shape shown in Fig. 2. This state is characterized by the plateau on the magnetization curve, shown in the Fig. 3. It means that the magnetic flux density inside the sample is unaffected by an increased external field. This perfect shielding should occur until the applied field reaches certain value of intensity, we call it second critical field for flux penetration. Then the vortices possessing magnetic structure enter into the sample. This phenomenon we named two-step flux penetration.

\section{ACKNOWLEDGEMENTS}

The author would like to thank P. Tekiel and K. Rogacki for helpful discussions. This work was supported by the State Committee for Scientific Research (KBN) within the Project No. 2 P03B 12519.

\section{References}

[1] For review see Superconductivity in Ternary Compounds, edited by M. B. Maple, and Ø. Fischer, Springer-Verlag, Berlin, 1982.

[2] M. B. Maple, Physica B 215, (1995) 110.

[3] K. Rogacki, E. Tjukanoff, and S. Jaakkola, Phys. Rev. B 64, (2001) 094520.

[4] T. Krzysztoń, Phys. Letters A 104, (1984) 225.

[5] H. Iwasaki, M. Ikebe, and Y. Muto, Phys. Rev. B 33, (1986) 4669. 
[6] T. Krzysztoń, J. Magn. Magn. Materials, 15-18, (1980) 1572.

[7] J. Zaretsky, C. Stassis, A. I. Goldman, P. C. Canfield, P. Dervenagas, B. K. Cho and D. C. Johnston, Phys. Rev., B 51, (1995) 678.

[8] S. K. Sinha, J. W. Lynn, T. E. Grigereit, Z. Hossain, L. C. Gupta, R. Nagarajan, C. Godard, Phys. Rev., B 51, (1995) 681.

[9] R. Szymczak, M. Baran, L. Gładczuk, H. Szymczak, Z. Drzazga, and A. Winiarska, Physica C254, (1995) 124.

[10] M. R. Eskildsen, A. B. Abrahamsen, D. Lopez, P. L. Gammel, D. J. Bishop, N. H Andersen, K. Mortensen, and P. C. Canfield, Phys. Rev. Lett., 86, (2001) 320 .

[11] T. Krzysztoń, G. Kozłowski, and P. Tekiel, Acta Phys. Polon, A 56, (1979) 49.

[12] V. G. Kogan, Phys. Letters,A 85, (1981) 298.

[13] J. R. Clem, in Proceedings of the 13th Conference on Low Temperature Physics (LT 13), vol. 3, Plenum-Press, New York 1974, p. 102.

[14] T. Krzysztoń, Phys. Letters A 190, (1994) 196.

[15] J. R. Clem and M. W. Coffey, Phys. Rev., B 42, (1990) 6209. 